\title{
COVID-19 Associated Acute Delirium
}

\author{
Authors \\ Dr Roy Sumeet Swapan, Dr Raghav Kapoor, Dr Goel Nikita
}

\begin{abstract}
Novel coronavirus disease (COVID-19), caused by SARS-CoV-2, which has been established as a worldwide pandemic, is now known to cause life-threatening respiratory, cardiovascular, and neurological complications. It's consequence on other organs is yet to be understood even after so many months have passed. The neuropsychiatric events following COVID-19, such as delirium, have been underreported and the etiology is poorly understood. The primary etiology of social stressor is believed to be the reason. A few documented reports have been published and hence there is no known specific management.

We are presenting a case report of an individual who presented with acute onset Delirium a few days after recovery from COVID infection. Wide range of investigations were done with no definite etiology or known pathophysiology. This phenomenon is self-resolving with no apparent consequence till date.
\end{abstract}

\section{Introduction}

Delirium is an acute mental status change characterized by abnormal and fluctuating attention. There is a disturbance in level of awareness and reduced ability to direct, focus, sustain, and shift attention. Recent cases of reactive psychosis in the context of the COVID-19 pandemic have emerged in literature, but less attention has been given to incident psychosis affecting patients with COVID-19.

Although a recent report documented cases of COVID-19-related psychosis in Madrid, this did not include a clinical description of affected patients $^{(1)}$. Ferrando et $a l^{(2)}$ described three cases of new-onset psychosis in patients with asymptomatic COVID-19, though two of these patients had a pre-existing psychiatric illness, and there were concerns for concurrent delirium. Here, we report a case of recovered COVID-19 related acute Delirium in a patient with no personal or family history of mental illness and briefly discuss the relevant literature on coronavirus-associated neuropsychiatric manifestations.

\section{Case Presentation}

A 47-year-old male was diagnosed COVID positive in the $3^{\text {rd }}$ week of April, 2021 and managed conservatively. He tested negative a week later and was discharged with full symptomatic recovery. One week later he was brought to the ER. He presented with new onset psychiatric symptoms such as hyperphagia, increased aggression and acute onset symptoms similar to that of a bipolar disorder. He had to be sedated and admitted as he was running about in the emergency.

He was afebrile with BP of $120 / 80 \mathrm{mmHg}$ and Pulse 84/minute. Despite his restless and 
aggressive behavior, he had no focal neurological deficit. A formal mental status examination was done that was normal for his level of education. The initial blood work up was unremarkable. We ordered a repeat COVID testing that came out negative. A second-tier work up including an autoimmune encephalitis and paraneoplastic panel was negative. TPO antibodies were within range. His MRI brain showed small focal areas of nonenhancing filling defects in superior sagittal sinus, possibly thrombosis which didn't explain the symptoms the patient showed. No significant brain parenchymal abnormality was however seen, EEG showed no epileptiform discharges. CSF examination showed no gross abnormality.

The patient was treated with enoxaparin $60 \mathrm{mg}$ twice daily for the CVT found on MRI. Tab Olanzapine $0.25 \mathrm{mg}$ twice a day and Tab Haloperidol $5 \mathrm{mg}$ as and when required for acute exacerbations was started. The patient symptoms started to decrease after 4 days of initiating the therapy. He was discharged with the same medications.

Follow-up after one week showed a drastic change in behavior and the anti-psychotic medications were tapered. He has had no symptoms ever since.

\section{Discussion}

The neuropsychiatric burden of COVID-19 is still unknown but is definitely significant ${ }^{(22)}$. A case series done in Madrid noted a number of potential cases of COVID-19 associated with psychosis in their hospital ${ }^{(1)}$. A case report of comorbid panic disorder and acute exacerbation of psychiatric symptoms in a person with associated with COVID 19 infection was also reported ${ }^{(3)}$. New psychiatric condition with no previous history of psychiatric disorder triggered by novel coronavirus was also reported in certain studies $^{(2,4)}$. The presented case also presented in same way, he didn't have any personal or family history of psychiatric symptoms. Further he did not have a history of substance abuse. He became symptomatic after 5 days of COVID negativity.
The exact pathology behind COVID infection and psychosis is not fully understood, but social stress surrounded by the COVID news induced social dilemma or viral mediated psychosis can be stated as a possible culprit ${ }^{(4)}$.

The virus infecting respiratory systems have been associated with psychiatric symptoms in the past with many other viruses. Many observational and case studies have been published stating the same. The viral infection of SARS (comes under coronavirus) and Middle Eastern respiratory Syndrome had documented cases of acute incidental psychosis in affected patients ${ }^{(5,6,7,8,9)}$. Association of psychiatric symptoms is also documented with influenza virus ${ }^{(10)}$. While the incident of psychosis is low, around $10 \%$ of individuals show symptoms ${ }^{(5,11,12)}$. Some reports suggest a correlation of psychiatric symptoms with higher steroid doses or severity of infection or personal or family history of psychiatric illness (8)

Although Steroid can be a suspected etiology in many cases the COVID-19 in itself can trigger psychosis through its neurotoxic effects or possibly a heightened immune response ${ }^{(4,7)}$. The evidence that antipsychotic such as haloperidol and chlorpromazine have shown antiviral effect and the COVID-19 has been isolated from the cental nervous system of the individual $(15,16,17)$. Points towards virus itself causing neurological signs and symptoms.

A study in Schizophrenia Bulletin journal ${ }^{(13)}$ showed that patient with IgG levels for two strains of coronavirus (HKU1 and NL63) showed significant psychotic symptoms when compared with controls, suggesting these two coronaviruses may be risk factors for neuropsychiatric illness.

"Profound inflammatory response to COVID-19 infection ("cytokine storm") is thought responsible for the severe, sometimes fatal, pulmonary and cardiac complications of the disease and has been postulated to produce neuropsychiatric symptoms through immunological mechanisms". ${ }^{(19,21)}$ Immune based mechanism is also postulated in few viral 
infections other than COVID-19 such as HIV $(19,20)$

The prevalence of this psychiatric incidences, severity and transientness of the symptoms prompts towards Thorough examination on suspected, confirmed and post COVID cases for any signs of psychiatric symptoms. Also, a review of all systems should be done at presentation with imaging to rule out any structural change in brain (4)

The studies for the exact management protocols for COVID-19 exaggerated psychosis is yet to be done. Hence, treatment of COVID included or post COVID psychosis should be focused on treating the underlying illness and managing the psychiatric symptoms with antipsychotics and benzodiazepines ${ }^{(14)}$.

We urge the biomedical community's attention to the needs of longitudinal monitoring of neuropsychiatric symptoms and neuroimmune status in individuals exposed to SARS-CoV-2 at different time points across the life course, including in utero, throughout childhood development, in adulthood, and in advanced age, to fully appreciate and mitigate the long-term deleterious impacts of COVID-19 on brain and behavior $^{(22)}$.

Conflicts of Interest: The authors have declared that no competing interests exist.

Funding: None

Consent for Publication: A Written Consent was obtained for the study.

\section{References}

1. Rentero D, Juanes A, Losada CP, et al. New-Onset psychosis in COVID-19 pandemic: a case series in Madrid. Psychiatry Res 2020;290:113097.

2. Ferrando SJ, Klepacz L, Lynch S, et al. COVID-19 psychosis: a potential new neuropsychiatric condition triggered by novel coronavirus infection and the inflammatory response? Psychosomatics
2020.

doi:10.1016/j.psym.2020.05.012.

[Epub ahead of print: 19 May 2020].

3. Galynker I, Ieronimo C, Perez-Acquino A, et al. Panic attacks with psychotic features. J Clin Psychiatry 1996;57:402-6.

4. Smith CM, Komisar JR, Mourad A, et al. BMJ Case Rep 2020;13:e236940. doi:10.1136/bcr-2020- 236940

5. Sheng B, Cheng SKW, Lau KK, et al. The effects of disease severity, use of corticosteroids and social factors on neuropsychiatric complaints in severe acute respiratory syndrome (SARS) patients at acute and convalescent phases. Eur Psychiatry 2005;20:236-42.

6. Lee DTS, Wing YK, Leung HCM, et al. Factors associated with psychosis among patients with severe acute respiratory syndrome: a case-control study. Clin Infect Dis 2004;39:1247-9

7. Brown ES, Chandler PA. Mood and cognitive changes during systemic corticosteroid therapy. Prim Care Companion J Clin Psychiatry 2001;3:1721.

8. Cheng SK-W, Tsang JS-K, Ku K-H, et al. Psychiatric complications in patients with severe acute respiratory syndrome (SARS) during the acute treatment phase: a series of 10 cases. $\mathrm{Br} \quad \mathrm{J}$ Psychiatry 2004;184:359-60.

9. Kim H-C, Yoo S-Y, Lee B-H, et al. Psychiatric findings in suspected and confirmed middle East respiratory syndrome patients Quarantined in hospital: a retrospective chart analysis. Psychiatry Investig2018;15:355-60.

10. Kępińska AP, Iyegbe CO, Vernon AC, et al. Schizophrenia and influenza at the centenary of the 1918-1919 Spanish influenza pandemic: mechanisms of psychosis risk. Front Psychiatry 2020;11:72

11. Kim H-C, Yoo S-Y, Lee B-H, et al. Psychiatric findings in suspected and 
confirmed middle East respiratory syndrome patients Quarantined in hospital: a retrospective chart analysis. Psychiatry Investig2018;15:355-60.

12. Mak IWC, Chu CM, Pan PC, et al. LongTerm psychiatric morbidities among SARS survivors. Gen Hosp Psychiatry 2009;31:318-26.

13. Severance EG, Dickerson FB, Viscidi RP, et al. Coronavirus immunoreactivity in individuals with a recent onset of psychotic symptoms. Schizophr Bull 2011;37:101-7.

14. Wilson MP, Pepper D, Currier GW, et al. The psychopharmacology of agitation: consensus statement of the American association for emergency psychiatry project beta psychopharmacology Workgroup. West J Emerg Med 2012; 13:26-34.

15. Troyer EA, Kohn JN, Hong S. Are we facing a crashing wave of neuropsychiatric sequelae of COVID-19? neuropsychiatric symptoms and potential immunologic mechanisms. Brain Behav Immun 2020;87:34-9

16. Gordon DE, Jang GM, Bouhaddou M, et al. A SARS-CoV-2 protein interaction map reveals targets for drug repurposing. Nature 2020;583:459-68.

17. Weston S, Coleman CM, Haupt R, et al. Broad anti-coronaviral activity of FDA approved drugs against SARS-CoV-2 in vitro and SARS-CoV in vivo. bioRxiv 2020.

18. The American Psychiatric Association. Practice guideline for the treatment of patients with schizophrenia. Available: https://www.psychiatry.org/psychiatrists/p ractice/ clinical-practice-guidelines2019 [Accessed 30 May 2020].
19. Ferrando, Stephen J et al. "COVID-19 Psychosis: A Potential New Neuropsychiatric Condition Triggered by Novel Coronavirus Infection and the Inflammatory Response?." Psychosomatics vol. 61,5 (2020): 551-555. doi:10.1016/j.psym.2020.05.012

20. Upthegrove R., Khandaker G.M. Cytokines, oxidative stress and cellular markers of inflammation in schizophrenia. Curr Top BehavNeurosci. 2019;44:49-66

21. Mehta P., Mcauley D.F., Brown M., Sanchez E., Tattersall R.S., Manson J.J. COVID-19: consider cytokine storm syndromes and immunosuppression. Lancet. 2020;395:1033-1034

22. Troyer, Emily A et al. "Are we facing a crashing wave of neuropsychiatric sequelae of COVID-19? Neuropsychiatric symptoms and potential immunologic mechanisms." Brain, behavior, and immunity vol. 87 (2020): 34-39. doi:10.1016/j.bbi.2020.04.027 